\title{
Ethical and scientific issues surrounding solid organ transplantation in HIV-positive patients: Absence of evidence is not evidence of absence
}

\author{
Timothy Christie $\mathrm{PhD}^{1,2,3}$, Bashir Jiwani MA ${ }^{4,5}$, Getnet Asrat $\mathrm{MD}^{1,2,3}$, Valentina Montessori MD FRCPC $1,2,3$, \\ Richard Mathias MD FRCPC ${ }^{2}$, Julio Montaner MD FRCPC $1,2,3$
}

\begin{abstract}
T Christie, B Jiwani, G Asrat, V Montessori, R Mathias, $\mathrm{J}$ Montaner. Ethical and scientific issues surrounding solid organ transplantation in HIV-positive patients: Absence of evidence is not evidence of absence. Can J Infect Dis Med Microbiol 2006;17(1):15-18.

End-stage liver disease is emerging as a leading cause of death among HIV-positive patients. Historically, an HIV diagnosis was a contraindication for a liver transplant; however, because of the efficacy of highly active antiretroviral therapy (HAART), HIV-positive patients have one-year, two-year, and three-year post-transplantation survival rates similar to that of HIV-negative patients. Based on this evidence, HIV-positive patients are now considered eligible for transplantation. However, newly emerging guidelines include the stipulation that HIV-positive patients must be on HAART to be placed on a waiting list for transplantation. The purpose of the present paper is to evaluate the scientific and ethical probity of requiring HIVpositive patients to be on HAART as a condition for being on a liver transplant waiting list. It is argued that the emphasis should be placed on the probability of post-transplantation HAART tolerance, and that concerns about pretransplantation HAART tolerance are of secondary importance.
\end{abstract}

Les enjeux éthiques et scientifiques entourant la transplantation d'organe chez les personnes séropositives au VIH : L'absence de données probantes ne signifie pas des données probantes d'absence

Key Words: Ethical decision-making; Ethics; Evidence-based medicine; Health policy; HIV-positive; Liver transplantation

$\mathrm{T}$

The purpose of the present paper is to evaluate the scientific and ethical probity of the British Columbia Transplant Society (BCTS) guidelines for liver transplantation in HIVpositive patients. As a result of highly active antiretroviral therapy (HAART), HIV-positive patients are now considered eligible for liver transplantation. However, newly emerging guidelines, such as those by the BCTS, include the stipulation that HIV-positive patients must be on HAART and have an undetectable viral load in order to be waitlisted for transplantation. We will argue two main points: first, that these requirements are not substantiated in the literature; and second, that in situations where research has not been done on a specific group, values should guide judgment rather than evidence. In this instance, the value should be to grant access to treatment until there is evidence to the contrary.

\section{BACKGROUND}

Since the introduction of HAART in 1996, rates of morbidity and death have fallen significantly among people infected with HIV (1). Patients are no longer dying from AIDS-related opportunistic infections. As a result, end-stage liver disease is now emerging as one of the leading causes of death among HIV-positive patients, which means that a liver transplant is the only treatment option for many patients $(2-6)$.

Before the introduction of HAART, an HIV diagnosis was a contraindication to liver transplantation. Typically, in the

\footnotetext{
${ }^{1}$ British Columbia Centre for Excellence in HIVIAIDS; ${ }^{2}$ University of British Columbia; ${ }^{3}$ Providence Health Care, Vancouver, British Columbia;

${ }^{4}$ University of Alberta, Edmonton, Alberta; ${ }^{5}$ Fraser Health Authority, Surrey, British Columbia

Correspondence and reprints: Dr Timothy Christie, BC Centre for Excellence in HIV/AIDS, St Paul's Hospital, \#613-1081 Burrard Street,

Vancouver, British Columbia V6Z 1Y6. Telephone 604-806-9263, fax 604-806-8464, e-mail tchristie@cfenet.ubc.ca

Received and accepted for publication November 10, 2005
} 
pre-HAART era, HIV-positive organ recipients died of infection soon after transplantation, and the survival outcomes for this population were lower than for the HIV-negative population (7). In addition, there were concerns about using immunosuppressive medications because they could precipitate opportunistic infections, accelerate progression to AIDS and decrease survival (8).

Internationally, outcomes have been reported for $51 \mathrm{HIV}$ positive patients who have received liver transplants since 1996 (3,8-16). The consensus is that HIV-positive liver transplant recipients currently have one-year, two-year and threeyear survival rates that are comparable with those of HIV-negative transplant recipients (17). Furthermore, posttransplantation survival rates in HIV-positive recipients coinfected with the hepatitis $C$ virus appear to be comparable to those of HIV-negative transplant recipients infected with hepatitis $\mathrm{C}$ virus $(3,9)$.

According to the Canadian Association of Transplantation, there are nine centres in Canada that perform liver transplants. Although no reports of transplantation in HIV-positive patients exist in the published literature, we have received anecdotal reports that one Canadian centre performed a liver transplant on an HIV-positive patient three years ago; however, this patient has since died. There are no published details about this specific patient, and it is unclear from these other centres in Canada whether they have guidelines for transplanting HIV-positive patients. Furthermore, most transplants of HIV-positive patients currently conducted in North America are being registered under research protocols as opposed to being provided within the treatment context. In reality, the details pertaining to the antiretroviral regimens used before and following transplantation, as well as adherence to and durability of these regimens, are not well described in the current literature. As a result, we have chosen to analyze the BCTS guidelines because they explicitly apply to liver transplantation in the treatment context (as opposed to the realm of research) and because they are the most clearly articulated treatment guidelines in Canada. We expect that other provinces will look to the BCTS for leadership on this issue.

It is important to delineate the distinction between treatment and research. Research is protocol driven, which means that the interventions that specific research subjects receive are determined by a strict research protocol (eg, inclusion criteria, exclusion criteria, duration of treatment). The treatment context is guided by the best interest principle, where collaboration between the patient and care provider(s) determines the interventions a particular patient receives. The BCTS guidelines apply to liver transplantation as a form of treatment (not as research). The ethical principles guiding treatment are, therefore, different from the principles guiding research. The BCTS guidelines read as follows:

- HIV-positive patients must fulfill all required criteria for liver transplant candidacy that non-HIV infected individuals must fulfill.

- HIV-positive patients must have an absolute CD4+ count of at least 200 cells/ $\mu \mathrm{L}$; however, in certain circumstances, provisions could be made to accommodate a lower CD4+ count.

- HIV-positive patients must be on HAART while they are on the waiting list (no minimum duration, HAART medications may be changed by HIV specialist at any time [ie, no specific duration or 'stable dose' required]).

- HIV-positive patients must have an undetectable HIV viral load at the time of transplantation.

The fact that the BCTS guidelines do not exclude a priori patients because of an HIV-diagnosis is highly appropriate because there appears to be no reason for excluding this patient population from the benefits of transplantation. In addition, despite restriction of a CD4+ cell count of at least 200 cells $/ \mu \mathrm{L}$, the allowance to accommodate patients with lower CD4+ cell counts on a case-by-case basis is also welcome for the same reason. However, the third and fourth guideline conditions, which require that the patient be on pretransplantation HAART and have an undetectable viral load, have the consequence of precluding many HIV-positive patients from being placed on the transplant waiting list and, hence, from receiving a liver transplant.

The problem is that many patients are unable to continue HAART because of liver toxicity and patients are often asked to consider liver transplantation in this setting so that they can resume HAART. Furthermore, patients who cannot tolerate HAART before receiving a liver transplant may not be able to achieve the undetectable viral load requirement. Similarly, such patients will most likely have a low CD4+ cell count, further jeopardizing their eligibility under the second guideline condition.

The BCTS guidelines correctly offer liver transplantation in HIV-positive patients as a form of treatment, rather than within the research context. However, because most of the research subjects that participated in these studies were on pretransplantation HAART, the guidelines incorrectly assume that pretransplantation HAART is part of the standard of care for this patient population. They further assume that because long-term outcomes are unknown in pretransplantation HAARTintolerant patients (because the issue has not been adequately investigated), these patients should not be eligible for transplantation. It is these specific assumptions that we wish to challenge.

\section{Argument 1}

We contend that simply because most research protocols specify pre- and post-transplantation HAART requirements, it does not logically follow that pretransplantation HAART is a necessary condition for a successful transplantation outcome. In fact, it is more likely that these protocols have pre- and post-transplantation HAART requirements so that somewhat homogeneous populations can be enrolled and compared. Most of the published studies were conducted as a proof of principle'; these studies found that solid organ transplantation could be performed safely and effectively in HIV-positive patients in the HAART era (18). The hypothesis tested was not whether pretransplantation HAART was a necessary condition for successful transplantation, but whether post-transplantation HAART could help improve transplantation outcomes in HIV-positive patients. The objectives were to show the contribution of post-transplantation HAART to improved outcomes in this setting.

The following quotation, from the inclusion criteria of two separate experimental protocols, clearly illustrates this issue. In fact, nearly identical language is used in these two distinct protocols (one in the United States at the University of California [18] and the other in Canada at McGill University); thus, only 
the McGill protocol will be quoted below. Specifically, the protocol requires that the research subject be on stable HAART for a minimum of 24 weeks before transplantation with the following caveat:

"Exceptions to this criterion may be allowed at the discretion of the local team (eg, an excellent transplant candidate in whom antiretroviral drugs cannot currently be tolerated because of liver disease) only if the HIV clinician on the team is confident that they can predict HIV suppression post-transplantation. This assessment should be made based on a thorough review of the patient's antiretroviral history, HIV-1 RNA levels while on medication, adherence and any resistance tests that are available. If there is any significant doubt about the ability to suppress viral replication post-transplantation, then the patient should not be enrolled under this criterion."

The essence of the above quotation is that post-transplantation HAART is far more important than the ability to tolerate HAART pretransplantation. This consideration was also implemented in a study by Roland and Stock (19). In this study, all transplant recipients were on pretransplantation HAART; however, the eligibility criteria specifically allowed subjects who were intolerant to pretransplantation HAART as long as they were expected to tolerate HAART after transplantation (19). Likewise, a recent paper reporting the Spanish experience (20) emphasized that the probability of complete viral suppression and immune reconstitution post-transplantation was more important than meeting the CD4+ cell count and viral load eligibility criteria.

More specifically, a small study by Ragni et al (10) explicitly considered the importance of pretransplantation HAART and viral load levels in terms of successful transplantation outcomes. They found that:

Survival was significantly poorer among transplant recipients with post-orthotopic liver transplantation (OLTX) antiretroviral intolerance $(\mathrm{P}=0.044)$ than among transplant recipients with pre-OLTX antiretroviral intolerance $(\mathrm{P}=0.239)$. In contrast, a pre-OLTX CD4+ cell count of less than 200 cells/ $\mu \mathrm{L}$ (compared with a cell count of at least 200 cells $/ \mu \mathrm{L}[\mathrm{P}=0.602])$ and a pre-OLTX HIV load of more than 400 copies $/ \mathrm{mL}$ (compared with a pre-OLTX HIV load of 400 copies $/ \mathrm{mL}$ or less $[\mathrm{P}=0.494]$ ) were not significantly associated with survival.

Therefore, we suggest that the BCTS guidelines may be based on a partial misinterpretation of the available evidence. The fallacy is in the conclusion, which states that pretransplantation HAART is required as part of the standard of care for this patient population because most of the research subjects in the relevant studies were on pretransplantation HAART. In the relevant literature, it has been unanimously found that post-transplantation HAART was necessary for a successful transplantation outcome. However, pretransplantation HAART was not considered a necessary condition for a successful outcome.

\section{Argument 2}

Arising from the above argument, it has further been argued that the absence of data pertaining to transplantation in patients who are intolerant to pretransplantation HAART is a sufficient reason to exclude them from receiving a liver transplant. The argument that a group should be denied access to treatment because research has not been done on them is rooted in faulty logic. A paucity of evidence does not automatically justify, from an ethical perspective, denying treatment.

Our allegiance to evidence-based treatment should not be used as an excuse to justify excluding groups of patients from accessing treatment simply because research has not been done on them. A common mistake when assessing evidence is to assume that 'no evidence of effect' is equal to 'evidence of no effect.' However, as Hartung et al (21) argued in 1983, the "absence of evidence is not evidence of absence". In the absence of evidence, a value judgment has to be made about whether to grant or deny access to treatment. Simply put, when decisions cannot be based on evidence, they must be based on values. We argue that in such situations, recognizing the important difference between treatment and research, the value should be to grant access to treatment until there is evidence to the contrary.

Although there is a paucity of evidence, there also appears to be a relative consensus among programs both within and outside Canada evaluating HIV-positive candidates for liver transplantation: HAART is not an absolute prerequisite for transplanting HIV-positive persons. Therefore, we are not discussing a complete absence of evidence because the balance of probabilities leans toward the conclusion that pretransplantation HAART is not a necessary condition for a successful outcome. Furthermore, the ethical argument lends credence to the fact that although the available evidence is limited, the underlying value should be to provide access to treatment until there is evidence to the contrary.

\section{Limitations}

The major limitation of the present analysis is our assumption that the motivation for these eligibility criteria is exclusively concerned with achieving good transplantation outcomes. The consequence of this assumption is that other objections to transplanting HIV-positive patients have not been considered in the present paper. For example, some critics may be concerned about allocating scarce resources to HIV-positive patients. Or as the following quotation (22) reveals, the undetectable viral load requirement may be motivated by health care provider considerations rather than transplantation outcomes:

"From the surgical perspective, concerns arise as to the risk to the surgical team in doing complex surgical procedures for patients with known HIV infection. There are obvious risks to the surgeons, anesthesiologists, and the many operating room staff who participate in liver replacement therapy."

These types of concerns may be important to discuss, but we have assumed that they are not what the pretransplantation HAART and undetectable viral load requirements are based on. If these eligibility criteria are motivated by something other than successful transplantation outcomes, then those arguments must be made available for scrutiny.

\section{CONCLUSION}

We argue that if HIV-positive patients satisfy all the criteria for liver transplantation candidacy that non-HIV-infected 
individuals satisfy, then the principle of justice requires that these patients be equally eligible for transplantation wait listing. The requirement that HIV-positive patients must be successfully treated on HAART to be accepted to a transplantation waiting list is not scientifically justified and, thus, does not meet the relevant ethical standard. Ultimately, it is unethical to tolerate unnecessary 'negative consequences'. The consequences of these eligibility criteria are both negative and unnecessary. We therefore propose that

\section{REFERENCES}

1. Wood E, Hogg RS, Harrigan PR, Montaner JS. When to initiate antiretroviral therapy in HIV-1-infected adults: A review for clinicians and patients. Lancet Infec Dis 2005;5:407-14.

2. Bica I, McGovern B, Dhar R, et al. Increasing mortality due to endstage liver disease in patients with human immunodeficiency virus infection. Clin Infect Dis 2001;32:492-7.

3. Neff G, Jayaweera D, Tzakis A. Liver transplantation for HIVinfected patients with end-stage liver disease. Curr Opin Organ Transplant 2002; 7:114-23.

4. Soriano V, Garcia-Samaniego J, Rodriguez-Rosado R, Gonzalez J, Pedreira J. Hepatitis C and HIV infection: Biological, clinical, and therapeutic implications. J Hepatol 1999;31(Suppl 1):119-23.

5. Sulkowski MS, Thomas DL, Chaisson RE, Moore RD. Hepatotoxicity associated with antiretroviral therapy in adults infected with human immunodeficiency virus and the role of hepatitis C or B virus infection. JAMA 2000;283:74-80.

6. Puoti M, Gargiulo F, Roldan EQ, et al. Liver damage and kinetics of hepatitis $\mathrm{C}$ virus and human immunodeficiency virus replication during the early phases of combination antiretroviral treatment. J Infect Dis 2000;181:2033-6.

7. Rubin RH, Jenkins RL, Shaw BW Jr, et al. The acquired immunodeficiency syndrome and transplantation. Transplantation 1987;44:1-4

8. Sayegh SE, Keller MJ, Huprikar S, Murphy B. Solid organ transplantation in HIV-infected recipients. Pediatr Transplant 2004;8:214-21.

9. Prachalias AA, Pozniak A, Taylor C, et al. Liver transplantation in adults coinfected with HIV. Transplantation 2001;72:1684-8.

10. Braitstein P. HIV infection and liver transplantation: A review of the literature. BC Medical Journal 2004;46:14-20.

11. Roland M, Carlson L, Ragni M, et al. Solid organ transplantation in HIV-infected recipients: A review of 53 cases in the HAART era. the central issue is the probability of a patient tolerating effective HAART post-transplantation, and that the requirement of pretransplantation HAART should be abandoned.

ACKNOWLEDGMENTS: The authors acknowledge the contribution of Anne Drummond, Medical Writer, BC Centre for Excellence in HIV/AIDS, for her editorial comments on the penultimate version of this paper.

Presented at the International AIDS Conference in Barcelona, Spain, 2002.

12. Ragni MV, Dodson SF, Hunt SC, Bontempo FA, Fung JJ. Liver transplantation in a hemophilia patient with acquired immunodeficiency syndrome. Blood 1999;93:1113-4.

13. Gow PJ, Mutimer D. Liver transplantation for an HIV-positive patient in the era of highly active antiretroviral therapy. AIDS 2001;15:291-2.

14. Sugawara Y, Makuuchi M, Morisawa Y, Kimura S, Tachikawa N, Oka S. [Living donor liver transplantation in a patient with HIV]. Nippon Rinsho 2002;60:803-6.

15. Kuo PC, Stock PG. Transplantation in the HIV+ patient. Am J Transplant 2001;1:13-7.

16. Tolan DJ, Davies MH, Millson CE. Fibrosing cholestatic hepatitis after liver transplantation in a patient with hepatitis C and HIV infection. N Engl J Med 2001;345:1781.

17. Ragni MV, Belle SH, Im K, et al. Survival of human immunodeficiency virus-infected liver transplant recipients. J Infect Dis 2003;188:1412-20.

18. Stock P, Roland M, Johnson L, et al. Protocol: Clinical, immunologic and pharmacologic consequences of solid organ transplantation in people with HIV infection. Cooperative clinical trials in adult transplantation, University of California, San Francisco, 2002.

19. Roland ME, Stock PG. Review of solid-organ transplantation in HIV-infected patients. Transplantation 2003;75:425-9.

20. Moreno S, Fortun J, Quereda C, et al. Liver transplantation in HIV-infected recipients. Liver Transpl 2005;11:76-81.

21. Hartung J, Cottrell JE, Giffin JP. Absence of evidence is not evidence of absence. Anesthesiology 1983:58:298-300.

22. Neff GW, Shire NJ, Rudich SM. Outcomes among patients with end-stage liver disease who are coinfected with HIV and hepatitis C virus. Clin Infect Dis 2005;41(Suppl 1):S50-5. 


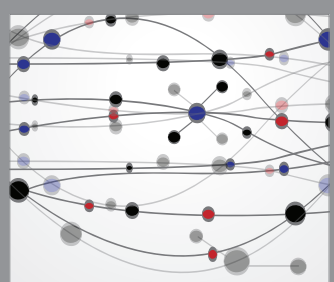

The Scientific World Journal
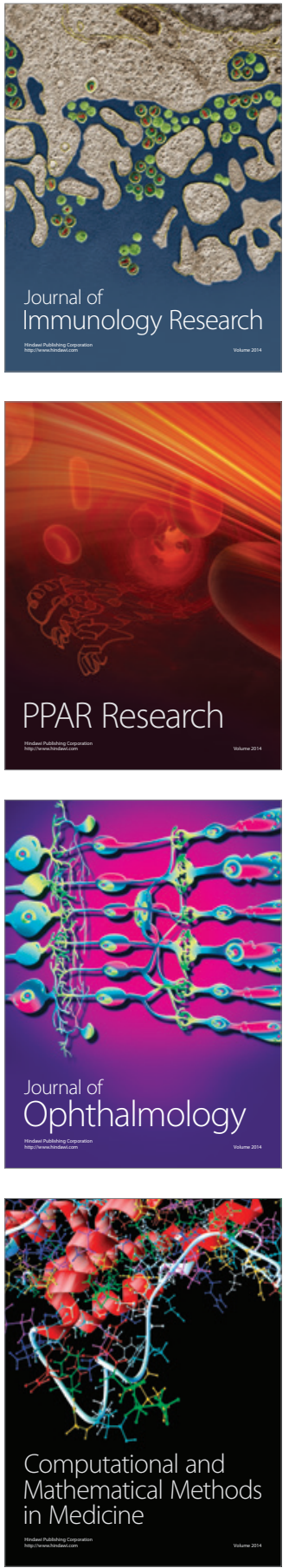

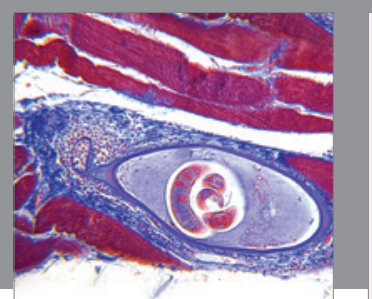

Gastroenterology Research and Practice

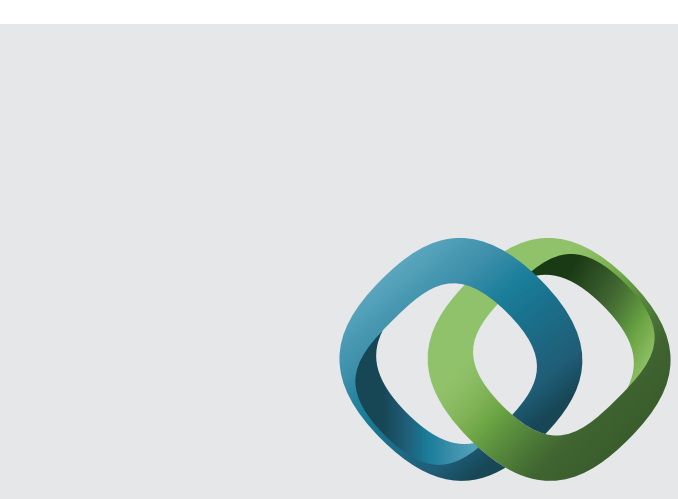

\section{Hindawi}

Submit your manuscripts at

http://www.hindawi.com
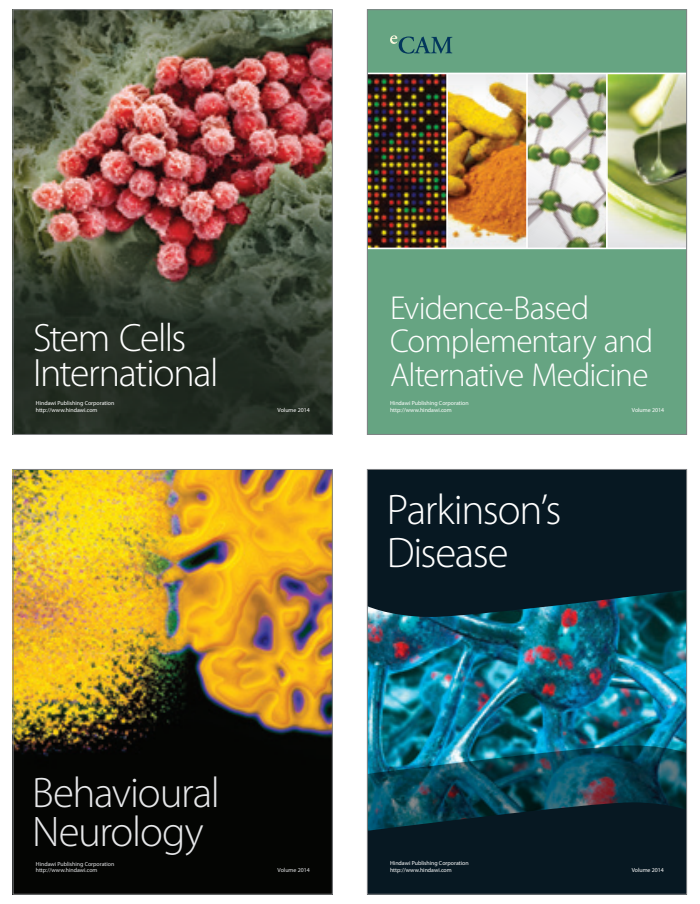
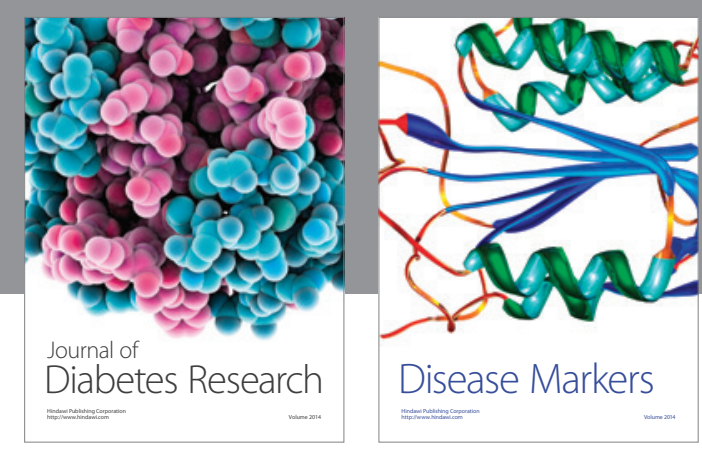

Disease Markers
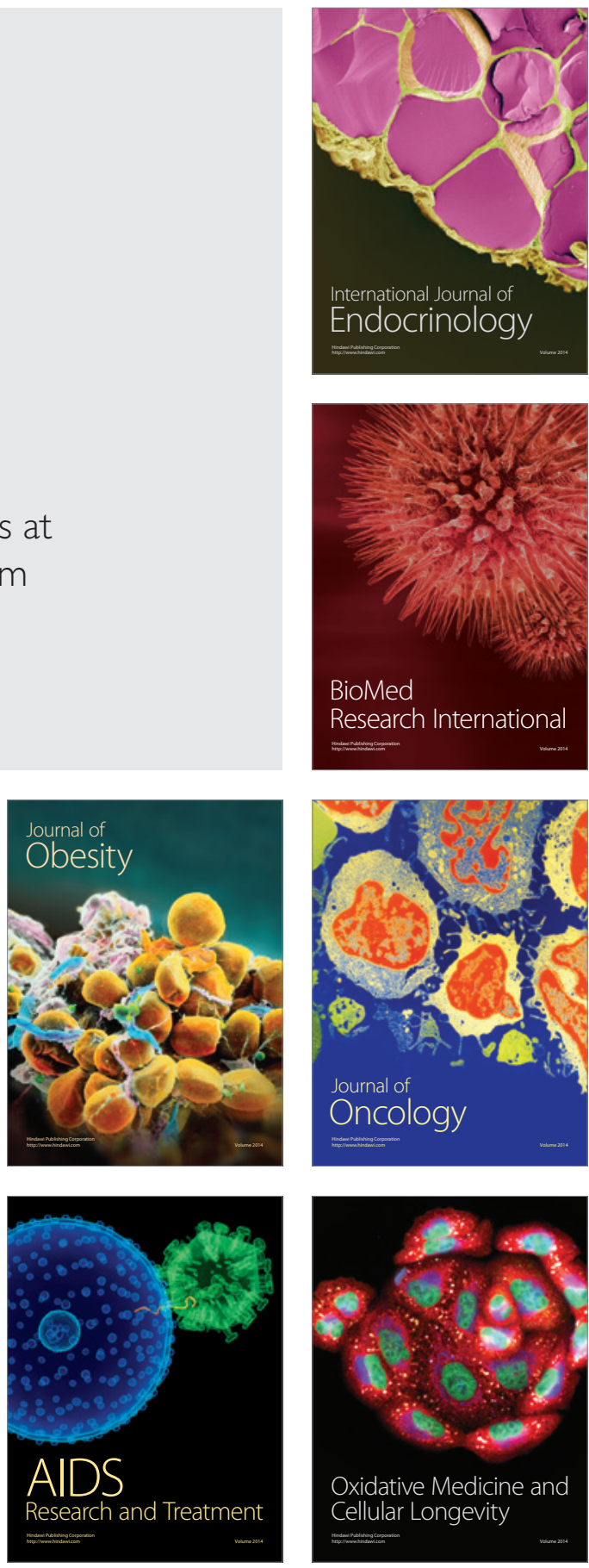